\title{
Hybrid Clustering of Long and Short-read for Improved Metagenome Assembly
}

Yakang Lu ${ }^{1, \dagger}$, Lizhen Shi ${ }^{2, \dagger}$, Marc W. Van Goethem ${ }^{3}$, Volkan Sevim ${ }^{4}$, Michael Mascagni ${ }^{2,5}$, Li Deng ${ }^{1, *}$ and Zhong Wang ${ }^{3,4,6, *}$

${ }^{1}$ School of Mechatronic Engineering and Automation, Shanghai University, Shanghai 200444, China, ${ }^{2}$ Department of Computer Science, Florida State University, Tallahassee, FL 32306, United States, ${ }^{3}$ Environmental Genomics and Systems Biology Division, Lawrence Berkeley National Laboratory, Berkeley, CA 94720, United States, ${ }^{4}$ Department of Energy Joint Genome Institute, Berkeley, CA 94720, California, United States, ${ }^{5}$ Applied and Computational Mathematics Division, National Institute of Standards and Technology, Gaithersburg, MD 20899, United States, ${ }^{6}$ School of Natural Sciences, University of California at Merced, Merced, CA 95343, United States

$\dagger$ These authors have contributed equally to this work.

Correspondence*:

Zhong Wang

zhongwang@lbl.gov

Li Deng

dengli@shu.edu.cn

\section{ABSTRACT}

Next-generation sequencing has enabled metagenomics, the study of the genomes of microorganisms sampled directly from the environment without cultivation. We previously developed a proof-of-concept, scalable metagenome clustering algorithm based on Apache Spark to cluster sequence reads according to their species of origin. To overcome its underclustering problem on short-read sequences, in this study we developed a new, two-step Label Propagation Algorithm (LPA) that first forms clusters of long reads and then recruits short reads to these clusters. Compared to alternative label propagation strategies, this hybrid clustering algorithm (hybrid-LPA) yields significantly larger read clusters without compromising cluster purity. We show that adding an extra clustering step before assembly leads to improved metagenome assemblies, predicting more complete genomes or gene clusters from a synthetic metagenome dataset and a real-world metagenome dataset, respectively. These results suggest that hybridLPA is a good alternative to current metagenome assembly practice by providing benefits in both scalability and accuracy on large metagenome datasets.

\section{Availability and implementation:}

https://bitbucket.org/zhong_wang/hybridlpa/src/master/.

Contact: zhongwang@lbl.gov

Keywords: Next-generation sequencing, hybrid metagenome clustering, Label Propagation Algorithm, metagenome assembly, PacBio sequencing, Oxford Nanopore sequencing 


\section{INTRODUCTION}

Metagenomics offers a fast track to directly study the microbial communities in their natural habitat without laboratory cultivation (Tyson et al., 2004, Hugenholtz and Tyson, 2008). Next-generation DNA sequencing (NGS) technologies have greatly expedited metagenomic discoveries, yielding deep insights into the composition, structure, and dynamics of complex microbial communities (Arumugam et al., 2011; Hess et al. 2011; $\mathrm{Xu}, 2006)$. Driven by the rapid development of NGS experimental technologies and modern, scalable metagenome assemblers, large numbers of individual microbial genomes can now be readily assembled from a single experiment or from meta-analyses constituting large cohorts of metagenomic datasets (Stewart et al., 2019; Parks et al., 2017; Nayfach et al., 2020). Currently, the Illumina Sequencing Platform is the predominant NGS platform for metagenome sequencing due to its high-throughput, lowcost, and high accuracy (average error rate $<1 \%$ ), despite that its short read length creates limitations on some downstream analysis tasks such as gene discovery (Wommack et al., 2008), read classification, or genome assembly (Breitwieser et al., 2019). To overcome these limitations, various strategies have been developed to either create synthetic long reads by assembly (Zimin et al., 2013) or experimentally (such as Moleculo, White et al. (2016)), but these methods bring additional experimental and/or computational costs.

Single-molecule, long-read sequencing technologies developed by Pacific Biosciences (PacBio, Eid et al. (2009)) and Oxford Nanopore Technologies (ONT, Schneider and Dekker (2012)) have been successfully applied to single-genome sequencing projects, yielding very high-quality genome assemblies from microbes to human (Chin et al., 2013; Koren and Phillippy, 2015; Logsdon et al., 2020; Sevim et al., 2019). These long reads, up to $100 \mathrm{~kb}$ in length, can effectively resolve large repeats or structural variations that pose challenges to short-read based assemblers. Long-read sequencing has not been widely adopted in metagenome sequencing, however, mainly because of two reasons. Firstly, PacBio and ONT long reads have error rates as high as 30\% (Eid et al., 2009, Schneider and Dekker, 2012). These errors, predominantly small insertions and deletions (indels), make the assembly process difficult and error-prone if they are not corrected. Secondly, compared with short-read sequencing, these technologies, when applied to complex metagenome projects, incur higher costs and lower throughput.

Recently, hybrid approaches have emerged to take advantage of the complementary characteristics of short and long-read sequencing technologies. Combining the high accuracy of short-read sequencing and the high read length of long-read sequencing, some genome assemblers such as Unicycler (Wick et al., 2017) and hybridSPAdes (Dmitry et al., 2016) showed promising results for single-genome assembly. However, most popular metagenome assemblers, including MEGAHIT (Li et al., 2015), MetaSPAdes (Nurk et al. 2017) and MetaHipmer (Hofmeyr et al., 2020), do not support hybrid assembly yet. The feasibility and potential benefits of a hybrid strategy in metagenome assembly were recently demonstrated by leveraging long reads for a second-round assembly of contigs from those metagenome assemblers (Bertrand et al. 2019).

We previously developed a scalable metagenome clustering tool called SpaRC (Shi et al., 2018, Li et al., 2020) based on Apache Spark. SpaRC can form pure and complete clusters with long-read sequencing technologies. However, it tends to produce a large number of small clusters on short-read datasets (underclustering) unless multiple samples from the same community are available. To illustrate this point, Table 1 shows the results of running SpaRC on two short-read datasets, each derived from a single sample of a synthetic microbial community: BMock12 (Sevim et al., 2019) and CAMI2 Simulated Toy Human Gut Metagenome (Sczyrba et al., 2017; Bremges and McHardy, 2018). In both experiments, SpaRC generated pure clusters but their completeness was very low. 
Table 1. Clustering Performance on Single-sample, Short-read, Synthetic Metagenome Datasets

\begin{tabular}{|c|c|c|c|c|}
\hline & \# reads & \# clusters & Median Purity & Median Completeness \\
\hline BMock12 & $10,517,108$ & 79,915 & 100 & 0.29 \\
\hline $\begin{array}{c}\text { CAMI2 Simulated } \\
\text { Toy Human }\end{array}$ & $296,027,232$ & $1,347,826$ & 100 & 11.62 \\
\hline
\end{tabular}

Motivated by the success of the above mentioned hybrid assemblers, in this study we explored a hybrid approach for metagenome read clustering to overcome the under-clustering problem of SpaRC. As SpaRC's core algorithm is based on the Label Propagation Algorithm (LPA), we first experimented three alternative label propagation strategies after long reads were added. Next, we explored the effect of using different proportions of long reads since long-read sequencing is relatively more costly. We also compared hybrid clustering performance of long-read datasets from both PacBio and ONT platforms. Finally, we evaluated the impact of hybrid clustering on downstream genome assembly and gene-cluster discovery performance, using a synthetic and a real-world metagenome dataset, respectively.

\section{MATERIALS AND METHODS}

\subsection{The Hybrid-LPA algorithm}

SpaRC uses Label Propagation Algorithm (LPA) originally proposed by Raghavan (Raghavan et al. 2007) to partition the read graph (Shi et al., 2018). Briefly, the algorithm begins by initializing each read with a unique label, followed by iteratively updating the label of each node to the label of the majority of its neighbors. After several iterations or until no further label propagation is possible, densely connected groups of reads are partitioned into clusters. LPA is capable of resolving genomes with shared reads and has near linear computational performance. SpaRC can be run at two different modes: "local mode" only cluster reads based on their overlap, while "global mode" further clusters the results from local mode based on multiple sample statistics (Li et al., 2020).

Here we explored three strategies for hybrid clustering with both long- and short-reads (Figure 11A):

- In the first "additive" strategy (S1), cluster labels can only propagate among long reads or among short reads, respectively. No propagation is allowed between long and short reads. This was done by running SpaRC at local clustering mode separately on the short-read and long-read datasets, and then combine the clustering results.

- In the second "mixed" strategy (S2), labels are allowed to propagate among both long and short reads indiscriminately: labels can propagate from long to long, short to short, long to short or vice versa. This was done by first combining the short- and long-read datasets, followed by running SpaRC at local clustering mode.

- In the third "long-then-short" strategy (S3), initially labels are only allowed to propagate among long reads. After all long reads finish updating their labels, their labels are allowed to propagate to short reads. This new algorithm, hereafter referred as hybrid-LPA, was implemented in both MPI and UPC++ in order to fit different HPC environments.

\subsection{Datasets and Data Preprocessing}

The BMock12 (Sevim et al., 2019) dataset was derived from a mock community that consists of 12 bacterial strains with genome sizes ranging 3.2 to $7.2 \mathrm{Mbp}$. One of the bacterial species in the set, $M$. coxensis, has a negligible number of reads in the dataset, therefore, BMock12 effectively contains 11 bacterial strains. The reads from BMock12 were downloaded from the NCBI Sequence Read Archive 
Table 2. Sequencing data statistics

\begin{tabular}{|c|c|c|c|c|}
\hline Dataset & Statistics & Illumina & ONT & PacBio \\
\hline \multirow{4}{*}{ BMock12 } & \#Reads & $211,448,444$ & 187,507 & 389,806 \\
\cline { 2 - 5 } & \#Bases & $63,384,840,109$ & $3,737,495,058$ & $2,583,337,248$ \\
\cline { 2 - 5 } & Max Length & 301 & 145,720 & 45,165 \\
\cline { 2 - 5 } & Min Length & 301 & 120 & 50 \\
\cline { 2 - 5 } & Avg Length & 301 & $19,932.6$ & $6,627.2$ \\
\cline { 2 - 5 } & Median Length & 301 & 17,900 & 5,800 \\
\cline { 2 - 5 } & Std_Dev & $/$ & $11,225.3$ & $4,283.2$ \\
\hline \multirow{5}{*}{ Biocrust } & \#Reads & $141,172,036$ & $/$ & $20,042,887$ \\
\cline { 2 - 5 } & \#Bases & $37,368,694,112$ & $/$ & $111,977,437,956$ \\
\cline { 2 - 5 } & Max Length & 301 & $/$ & 138,853 \\
\cline { 2 - 5 } & Min Length & 35 & $/$ & 550 \\
\cline { 2 - 5 } & Avg Length & 264.7 & $/$ & 5427 \\
\cline { 2 - 5 } & Median Length & 301 & $/$ & $3,324.0$ \\
\cline { 2 - 5 } & Std_Dev & 51.5 & $/$ & \\
\hline
\end{tabular}

100 (SRA) using accessions SRX4901586 (ONT), SRX4901584 (PacBio set 1), SRX4901585 (PacBio set 2), and SRX4901583 (Illumina). Table 2 lists the statistics of these datasets. The Illumina short-read dataset from this community was pair-end sequenced at $150 \mathrm{bp}$. The two ends were concatenated by an "N" (resulting a 301bp fragment) before being fed into SpaRC. In this paper, we took 5\% of the reads from the original dataset to conduct the experiments.

The Biocrust dataset was derived from a biological soil crust sample collected from Moab, UT, USA. Biocrusts are specialized microbial communities consisting of primary producers, such as cyanobacteria, mosses, and lichens, and associated heterotrophs. They are aggregated organosedimentary communities that colonize and stabilize the soil surfaces of arid environments, preventing soil erosion and promoting nutrient status by fixing both atmospheric carbon and nitrogen (Van Goethem et al., 2021). The two ends of a Illumina short-read pair are 151 and 150bp. The two ends were merged by BBMerge (Bushnell et al., 2017). The merged Illumina reads, as well as the PacBio reads, were masked for low-complexity sequences by BBDuk using default parameters (sourceforge. net/projects/bomap/). The resulting fragments were used as input for SpaRC.

\subsection{Running SpaRC and Hybrid-LPA}

Small-scale experiments in this work were performed on the Amazon Web Services (AWS) Cloud. Apache Spark (ver 2.3.1) services and Hadoop (ver 2.8.4) are provided by the Elastic MapReduce (EMR) on AWS. Specifically, we first used SpaRC to generate read graphs (EMR, emr-5.17.0). Then we used one node (r4.16xlarge) with $64 \mathrm{CPU}$ cores and 488GB memory to run hybrid-LPA. On the EMR cluster, one node is used as the master and all other nodes (r4.2xlarge) are used as workers. Depending on the size of the input datasets, various number of workers are used (20 workers for BMock12 and 200 for the Biocrust dataset).

Large-scale experiments were performed on Berkeley Lab's High-performance Computing system (Lawrencium, https://sites.google.com/a/lbl.gov/hpc/) and Department of Energy's National Energy Research Scientific Computing Center (NERSC, https://www. nersc.gov/). In these environments, SpaRC jobs were run on standalone Spark clusters created on-demand. Specifically, 600 Cori KNL nodes (each has 68 physical cores and 96 GB of memory) on NERSC were used for the Biocrust dataset. 


\section{RESULTS} number of BGCs). purity was calculated.

\subsection{Metagenome Assembly and Binning, Biosynthetic Gene Cluster Prediction}

For the BMock12 dataset, each cluster from the hybrid-LPA output was assembled by metaSPAdes (ver 3.13.1) using default parameters (Nurk et al., 2017). Contigs from all clusters were combined for binning with MetaBAT 2 (Kang et al., 2019) using default parameters. In the assembly-only method, raw reads were assembled with metaSPAdes followed by binning with MetaBAT 2. MetaQuast (version 5.0.2) was used to evaluate assembly quality for both two methods (Mikheenko et al., 2016).

From the assembled biocrust metagenomes (performed using metaSPAdes, Canu (Koren et al., 2017) and metaFlye (Kolmogorov et al., 2020), providing 3 assemblies) we deduplicated the contigs using BB-Dedup using default parameters(sourceforge.net/projects/bbmap/) to only include unique sequences by removing redundant contigs. All contigs longer than $5 \mathrm{~kb}$ were retained for secondary metabolite production using antiSMASH v5.2.0 under strict settings to preclude the detection of false-positives (Blin et al. 2019). Here, biosynthetic gene clusters (BGCs) were retained if they were longer than $5 \mathrm{~kb}$ after manual inspection of the domain architecture. Finally, we compared the quantity of unique BGCs detected when clustering-then-assembling to assembly-only (metaFlye assembly only, as it produced the largest

\subsection{Long reads increase clustering performance}

To test whether or not combining long reads with short reads improved clustering performance, we designed three strategies (Materials and Methods) to include long reads in SpaRC's LPA step (Figure 1A). We ran the three strategies on the synthetic BMock12 dataset (Materials and Methods) with 12 known genomes and used three metrics to measure read clustering performance: read cluster size (number of reads in a cluster), purity (percent of reads from the predominant genome in a cluster) and completeness (percent of reads from the predominant genome in a cluster). For these experiments, we used Illumina short reads and ONT long reads. Since we aimed at exploring how the long reads help with short reads clustering, these metrics were calculated based on short reads only. In addition, as we did not expect SpaRC to distinguish different strains of the same species, strain-level differences were ignored when clustering

Figure $1 \mathrm{~B}$ illustrates the cluster size comparison between these different label propagation strategies. The additive strategy (S1) produces many small clusters. Clusters formed from the mixed strategy (S2) showed a bi-modal size distribution, characterized by the presence of many larger clusters and small clusters. In contrast, the long-then-short strategy (S3) only produces a small number of clusters, most of them are very large. These strategies resulted in similar numbers of short reads in clusters (Table 3). However, the number of clusters was reduced from 85,398 (S1) to 136 (S3), while the mean cluster size was increased from 125.3 (S1) to 75,749.1 (S3). Consequently, the median completeness was increased from $0.25 \%$ (S1) to $79.42 \%$ (S3). As shown in Figure $1 \mathrm{C}$, this increase of genome completeness by S3 was reflected in that the majority of clusters having better completeness, a significant shift from the other two strategies. These improvements in cluster size and completeness did not come with a decreased clustering purity, with a median purity $100 \%$ and a mean purity $99.65 \%$ (Figure $1 \mathrm{C}$ ). Clustering performance of the long-then-short strategy also outperforms the mixed strategy in terms of completeness, number of clusters, and cluster size.

These results suggest that long reads can greatly improve metagenome read clustering performance and that the hybrid clustering strategy presented here is an effective way to solve the under-clustering problem with metagenomic short reads. 
A

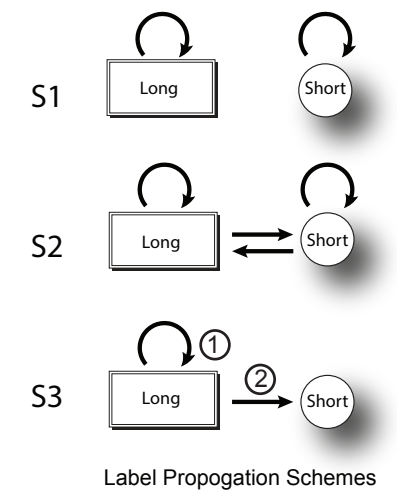

B
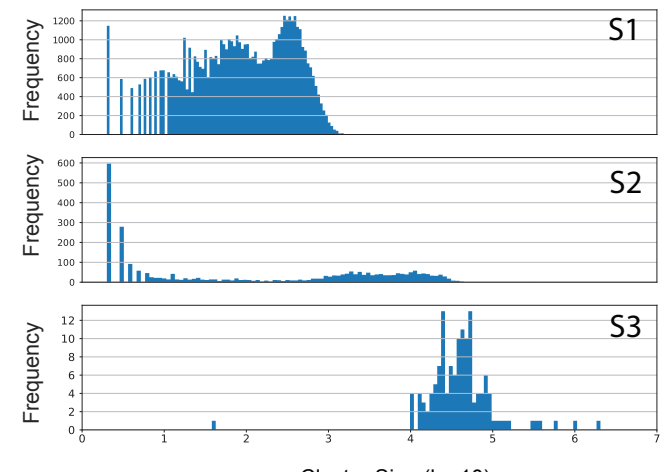

Cluster Size $(\log 10)$

C

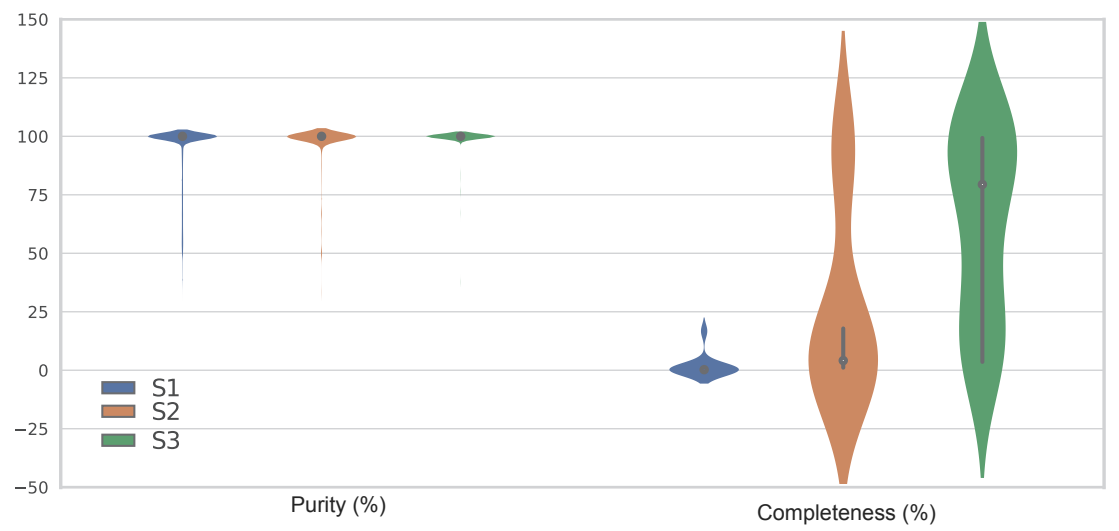

Figure 1. (A) Three alternative clustering strategies for hybrid-LPA. (S1) "Additive" strategy: clustering labels can only propagate among long reads or among short reads, respectively. No propagation was allowed between long and short reads. (S2) "Mixed" strategy: labels can be propagated among both long and short reads indiscriminately. (S3) "Long-then-short" strategy: in the first step, labels were only allowed to propagate among long reads, then they were propagated to short reads. No propagation was allowed among short reads. (B) A comparison of three label propagation strategies on cluster size improvement on the BMock12 dataset. The number of clusters ( $Y$-axis) at each cluster size in $\log 10$ (X-axis), from top to bottom: S1, S2, S3. (C) A comparison of three label propagation strategies on the purity and completeness of clusters on the BMock12 dataset. Violin plots of purity and completeness distributions are shown in percentage $(Y$-axis).

Table 3. Clustering performance comparison between the three LPA strategies

\begin{tabular}{|l|c|c|c|c|c|c|c|}
\hline & \#clusters & \#reads & $\begin{array}{c}\text { \% of reads } \\
\text { clustered }\end{array}$ & $\begin{array}{c}\text { mean cluster } \\
\text { size }\end{array}$ & $\begin{array}{c}\text { median } \\
\text { completeness }\end{array}$ & $\begin{array}{c}\text { median } \\
\text { purity }\end{array}$ & $\begin{array}{c}\text { mean } \\
\text { purity }\end{array}$ \\
\hline S1 & 85,398 & $10,312,376$ & 96.38 & 125.3 & 0.25 & 100 & 97.90 \\
\hline S2 & 15,145 & $10,312,376$ & 96.38 & 706.5 & 4.13 & 100 & 94.62 \\
\hline S3 & 136 & $10,298,222$ & 96.25 & $75,749.1$ & 79.42 & 100 & 99.65 \\
\hline
\end{tabular}

Although long reads greatly reduce the under-clustering problem in the above experiment, they did not solve the over-clustering problem, as some clusters contain reads from different genomes. Among the top 20 largest clusters, 17 of them are pure clusters at the species level (Table 4). The biggest cluster, consisting 2 million reads ( $20 \%$ of the clustered reads), mixed reads from two different closely-related species (Marinobacter sp.1 and Marinobacter sp.8) of the same genus (Marinobacter), owing to the fact 
bioRxiv preprint doi: https://doi org/10.1101/2021.01 25.428115: this version posted January 26.2021 . The copyright holder for this preprint (which was not certified by peer review) is the author/funder. This article is a US Government work. It is not subject to copyright under 17 USC 105 and is also made available for use under a CCO license.

Table 4. Top 20 cluster size and composition

\begin{tabular}{|c|c|c|c|}
\hline cluster \# & \# reads in cluster & $\begin{array}{l}\text { percentage of the total } \\
\text { clustered reads }(\%)\end{array}$ & cluster composition (species level) \\
\hline 1 & $2,053,694$ & 19.54 & $\begin{array}{l}\text { Marinobacter sp.8: } 76 \% \text {, } \\
\text { Marinobacter sp.1: } 24 \%\end{array}$ \\
\hline 2 & 978,753 & 9.31 & $\begin{array}{l}\text { Cohaesibacter sp.: } 38 \%, \\
\text { Thioclava sp.: } 30 \%, \\
\text { Propionibact. b.: } 12 \%, \\
\text { M. echinofusca: } 11 \%, \\
\text { M. echinaurantiaca: } 9 \%\end{array}$ \\
\hline 3 & 583,575 & 5.55 & $\begin{array}{c}\text { Halomonas sp. : 67\%, } \\
\text { Psychrobacter sp.6: } 15 \%, \\
\text { Marinobacter sp.8: } 7 \%, \\
\text { Muricauda sp.: } 7 \%, \\
\text { others: } 4 \%\end{array}$ \\
\hline 4 & 396,548 & 3.77 & Halomonas sp. : $100 \%$ \\
\hline 5 & 350,579 & 3.34 & Cohaesibacter sp.: $100 \%$ \\
\hline 6 & 310,604 & 2.95 & Halomonas sp.: $100 \%$ \\
\hline 7 & 162,263 & 1.54 & Psychrobacter sp.6: $100 \%$ \\
\hline 8 & 141,331 & 1.34 & Halomonas sp.: $100 \%$ \\
\hline 9 & 127,583 & 1.21 & Halomonas sp.: $100 \%$ \\
\hline 10 & 118,194 & 1.12 & Halomonas sp.: $100 \%$ \\
\hline 11 & 101,535 & 0.97 & Psychrobacter sp.6: $100 \%$ \\
\hline 12 & 96,011 & 0.91 & Halomonas sp. : $100 \%$ \\
\hline 13 & 95,349 & 0.91 & Halomonas sp.: $100 \%$ \\
\hline 14 & 89,545 & 0.85 & Halomonas sp. : $100 \%$ \\
\hline 15 & 88,730 & 0.84 & Halomonas sp.: $100 \%$ \\
\hline 16 & 84,861 & 0.81 & Halomonas sp. : $100 \%$ \\
\hline 17 & 84,394 & 0.80 & Psychrobacter sp.6: $100 \%$ \\
\hline 18 & 82,854 & 0.79 & Halomonas sp. : $100 \%$ \\
\hline 19 & 82,168 & 0.78 & Halomonas sp. : $100 \%$ \\
\hline 20 & 82,083 & 0.78 & Halomonas sp.: $100 \%$ \\
\hline others & $4,401,089$ & 41.87 & 1 \\
\hline total & $10,511,743$ & 100.00 & T \\
\hline
\end{tabular}

that these species have an average nucleotide identity (ANI) of 78.1\%, and they share 105,617 common 31-mers, making them difficult to be distinguished (Supplemental Table S1). As expected, the clustering algorithm could not distinguish closely related strains of the same species, such as Halomonas sp. HL-4 and Halomonas sp. HL-93, with 3,126,579 shared 31-mers and an ANI of 98.5\%. This pair of genomes spread 14 of the top 20 clusters. Different species with a large number of shared k-mers could also get clustered together, as the second and third largest clusters each contain multiple genomes. Some of these genomes are related, but some are not clearly indicating an over-clustering problem.

\subsection{Small amounts of long-read data sufficiently improve clustering}

As long-read sequencing technologies have higher cost and lower throughput, we tested whether or not limited numbers of long reads can help short-read metagenome clustering. In the following experiments done on the BMock12 dataset, we gradually increased the amount of ONT reads added to Illumina reads and compared the hybrid clustering performance.

As shown in Figure 2, adding just $1 \%$ ONT reads already produces a pronounced effect, increasing the mean cluster size to over 50,000 reads. Except for some variations when below 10\% of the ONT reads were added, adding more ONT reads increases the mean cluster size, even though the increase gets smaller. The 


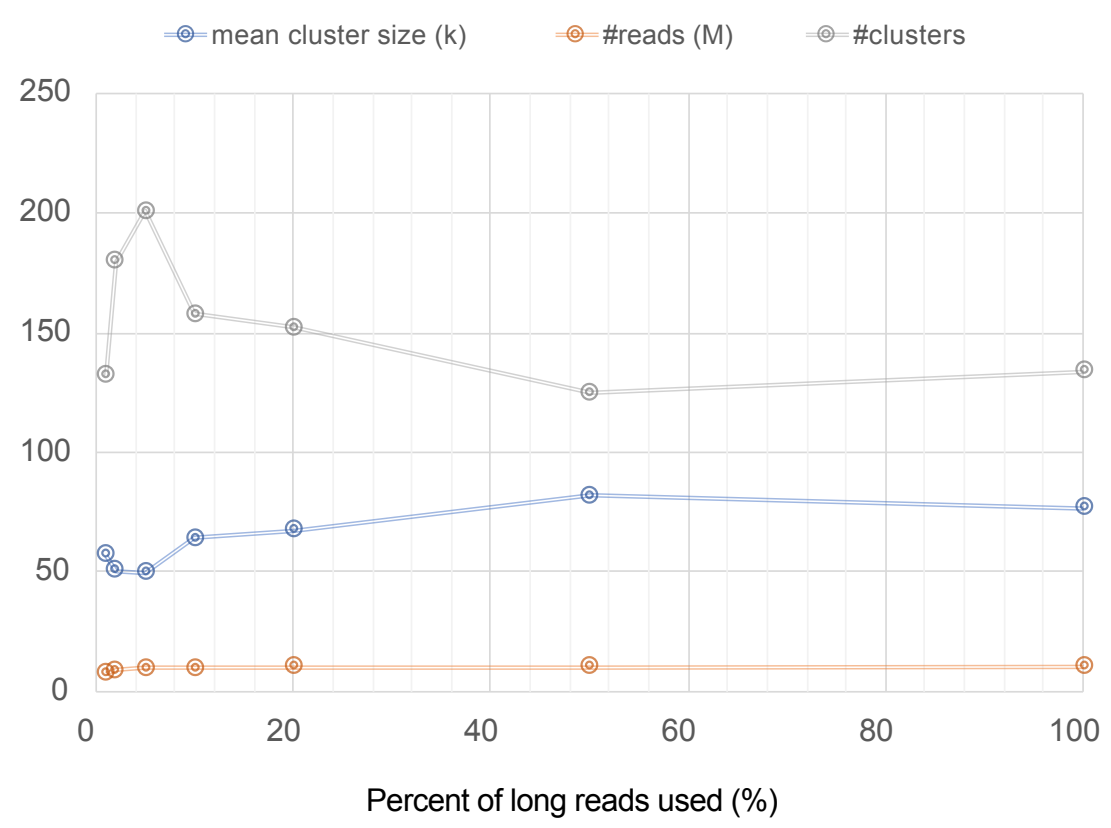

Figure 2. The effect of different amounts of ONT reads added to Illumina short reads on cluster size: the number of clusters (blue line), the number of reads being clustered in millions ( $\mathrm{M}$, grey line), and the mean cluster size in thousands (K, orange line) vary as different percentages of ONT long reads are added $(X$-axis)

total number of clusters first rises, then steadily falls after 5\% ONT reads. The total number of clustered short reads remains largely unchanged. As we added more long reads ( $>10 \%$ of total), the number of reads clustered, the number of clusters formed, and the mean cluster size all become stable. These results suggest a small fraction of long reads can significantly improve short read clustering, and the hybrid clustering approach could be a cost-effective metagenome clustering method.

\subsection{Read length, not the sequencing platform, has a major impact on the cluster size}

In theory, longer read lengths should increase the clustering performance, as their ability to bridge short reads gets better with length. To test this hypothesis, we added shorter PacBio reads from the same BMock12 dataset and compared the results to the above obtained from ONT reads. The read length distribution of ONT and PacBio reads is shown in Figure $3 \mathrm{~A}$.

As expected, ONT read hybrid clustering gave much better results than those from PacBio reads. The number of clusters from the ONT experiment is 136, while the PacBio produced 1,502 clusters (Figure $3 \mathrm{~B}$ ). The corresponding genome completeness metrics were measured at $79.42 \%$ and $7.09 \%$ for ONT and PacBio, respectively. The size of the clusters produced by adding ONT reads is much larger than that of PacBio reads (Figure 3C). To investigate whether this difference is caused by different platforms rather than by different read lengths, we trimmed the ONT reads so that they have the same length distribution as the PacBio reads (Figure 3A) and then repeated the experiment. The number of clusters became 1,149 by adding the trimmed ONT reads, which is very similar to the results obtained from the PacBio reads. And the genome completeness for trimmed ONT was reduced to $10.91 \%$. The cluster size distribution is also comparable to the results of PacBio experiment. In all three experiments, the median purity metrics of the clusters are comparable, ranging from $97.73 \%-100 \%$. These results confirmed that the read length, rather than the long-read sequencing platform, has a major impact on clustering performance. 

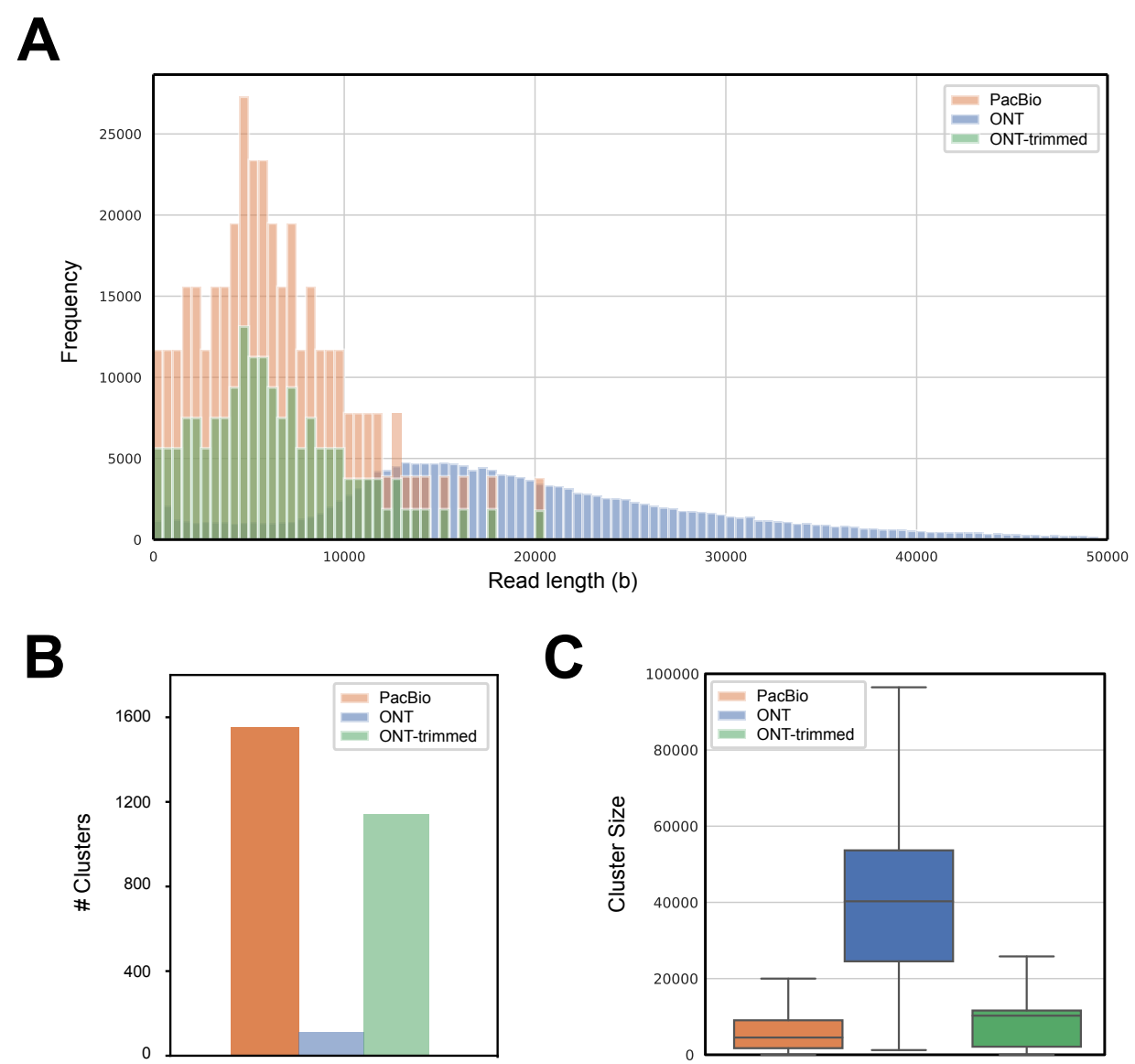

Figure 3. The dependency of hybrid clustering performance on read length. (A) Read length distribution of PacBio (orange), ONT (blue) and trimmed ONT (green) reads to match PacBio read length distribution in BMock12: the read length $(X$-axis) is plotted with its respective number of reads $(Y$-axis) for ONT and PacBio sequencing platforms. (B) The number of clusters from hybrid-LPA using PacBio, before and after trimming ONT. The number of clusters is much smaller in ONT than PacBio but becomes comparable after the trimming. (C) Box plots of cluster size from hybrid-LPA using PacBio, before and after trimming ONT. The cluster size (Y-axis) after trimming ONT read length is comparable to PacBio, both are much smaller than ONT.

\subsection{Hybrid clustering improves downstream metagenome assembly and gene cluster discovery}

To investigate whether or not the improved clustering results produced by hybrid clustering can translate into better downstream applications, we used two common scenarios as examples. First, on the BMock12 dataset where the set of genomes are known, we asked whether or not hybrid clustering produces better metagenome-assembled-genomes (MAGs). Second, we used a real-world Biocrust metagenome dataset without known references (Materials and Methods), and asked whether or not hybrid clustering could produce more predicted biosynthetic gene clusters (BGCs), locally clustered genes that together encode a biosynthetic pathway for the production of secondary metabolites (Medema et al., 2015). In both cases we wanted to compare the results to metagenome assembly with hybrid clustering (hereafter we refer as "SpaRC-hybrid") and without ("Assembly"). The steps in this two methods are otherwise identical except in the SpaRC-hybrid the assembly was done on the clusters instead of on raw reads. A schematic view of the two methods is shown in Figure $4 \mathrm{~A}$. 
For the BMock12 dataset, the quality of genome bins were evaluated using Quast (Gurevich et al., 2013). Quast produces many metrics, here we focused on two assembly-related ones: the percent of genome coverage that measures the extent that a genome bin covers a reference genome, and percent of correctly assembled that measures the percent of assemblies aligned to references without any mis-assemblies (Figure 4B). Using $80 \%$ genome fractions and $90 \%$ correctness as cut-offs, the SpaRC-hybrid method produces 8 good genomes while the Assembly method only produced 4, supporting the notion that hybrid clustering improves downstream genome assembly. The full Quast report is available in Supplemental Table S2. Other differences between these two methods we noticed include SpaRC-hybrid producing much smaller N50s, higher rates of mismatches and small indels. These observations suggest the under-clustering problem still exists to some extent, so that the assemblers do not have sufficient read coverage for correcting the errors in long reads, or producing good contiguity.

For the Biocrust dataset, we used the ability to discover unique Biosynthetic Gene Clusters (BGCs) as a metric to test the benefit of hybrid-LPA over the Assembly method without prior clustering (Materials and Methods). Overall, the SpaRC-hybrid method predicted more BGCs than the Assembly method alone (Figure 4C). MetaFlye assembly derived from SpaRC-hybrid clusters gave 5,458 unique BGCs, considerably more than those from the Assembly approach (2,988 BGCs). In almost every category SpaRC-hybrid predicted more BGCs, with the most pronounced difference in Non-ribosomal peptides, a common and important class of secondary metabolites encoded by multidomain non-ribosomal peptide synthetases (NRPS). A complete list of the counts are available in Supplemental Table S3. The hybrid approach also predicted more complete gene clusters (i.e., it is not truncated on either of the contig edges) than the assembly-only approach, 1,100 vs 712 (Van Goethem et al., 2021). The longest NRPS is novel (based on sequence similarity to the entire NCBI nr database) and is a full-length gene cluster of 79,925 bp.

We made similar observations when we assembled the clusters using CANU instead of MetaFlye (Supplemental Table S3), suggesting hybrid clustering by SpaRC-LPA can benefit downstream assemblers in general.

\section{DISCUSSION}

In this work, we developed a new scalable algorithm, SpaRC-hybrid to incorporate long reads into metagenome read clustering. We showed that the hybrid clustering method can reduce the under-clustering problem in clustering experiments with only short reads. We also demonstrated that the read length, rather than the sequencing technologies, has a big impact on the clustering performance. Furthermore, improved clustering results can greatly augment downstream metagenome assembly or gene cluster discovery.

While SpaRC-hybrid can effectively leverage long reads to reduce the under-clustering problem in short reads, it does not reduce over-clustering problems, where similar genomes, or genomes sharing large genetic elements (horizontally transferred genes, very closely-related homologs, mobile elements, etc.) are clustered together. Given that SpaRC-hybrid uses long reads to build the initial read graph, it should alleviate the problem to some extent at this stage. However, over-clustering can still happen at the short-read recruitment stage. Using stringent read overlapping criteria may reduce the problem, but this may come with a cost of under-clustering and loss in sensitivity. In complex real-world metagenome datasets, this is unlikely to be a major drawback, as the overall complexity within a cluster could be greatly reduced compared to the original dataset. We may not be able to completely deconvolute a large, complex metagenome into single genomes, but can effectively partition into many simpler metagenomes. With the decreasing cost and increasing throughput of long-read sequencing, ultimately we may have to use only long reads for metagenome clustering to overcome the over-clustering problem. 
bioRxiv preprint doi: https://doi.org/10.1101/2021.01.25.428115; this version posted January 26, 2021. The copyright holder for this preprint (which was not certified by peer review) is the author/funder. This article is a US Government work. It is not subject to copyright under 17 USC 105 and is also made available for use under a CCO license.

A

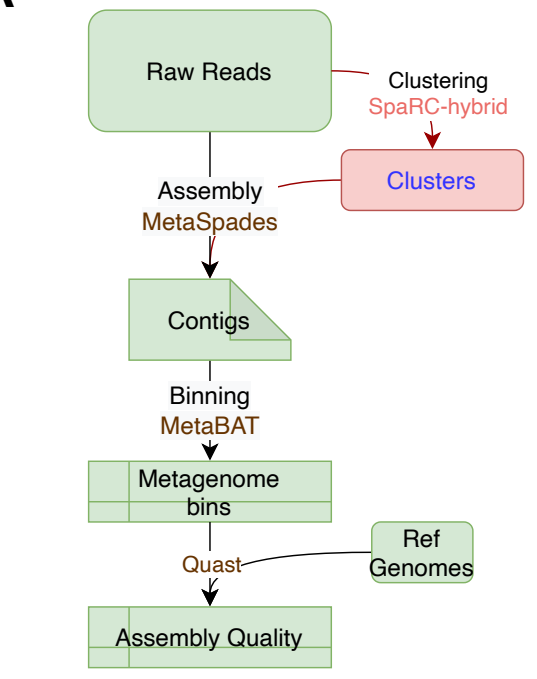

Assembly vs SpaRC_hybrid
B

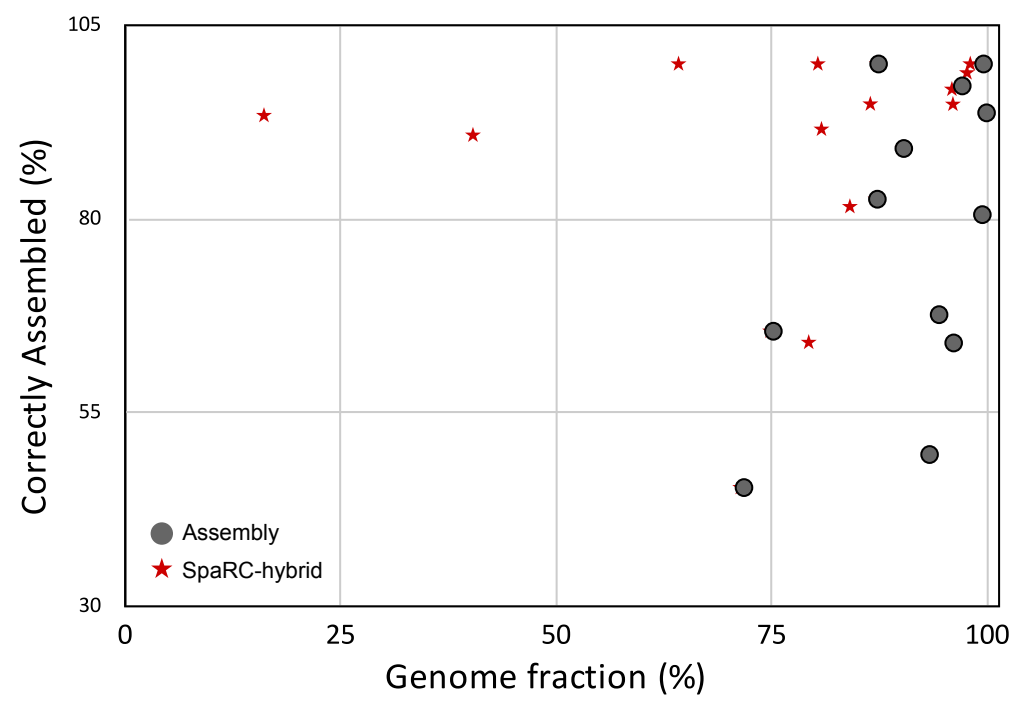

\section{C}

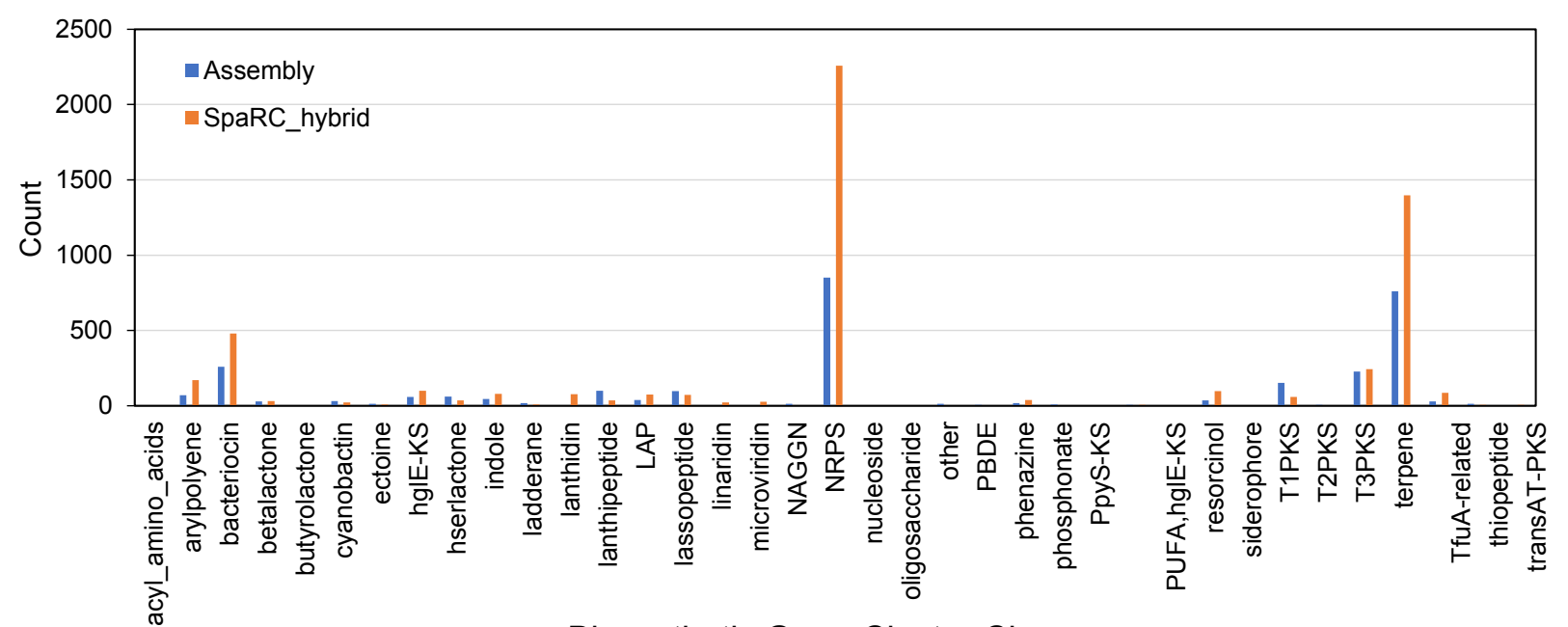

Biosynthetic Gene Cluster Class

Figure 4. (A) A schematic view of metagenome hybrid assembly methods. Default "Assembly" method first assembles the raw reads (short and long) using an assembler (such as MetaSpades Nurk et al. (2017)), and then bins the resulting contigs into metagenome bins by a binner (such as MetaBAT Kang et al. (2015)). If reference genomes are available, the quality of the bins can be evaluated by Quast. The "SpaRC-hybrid" method first clusters the raw reads into clusters, then assembles the clusters into contigs, followed by the same procedures as the Assembly approach. (B) A comparison of assembled genome quality between the Assembly and SpaRC-hybrid approach on the BMock12 dataset. Two metrics measured by Quast, Genome Fraction percentage $(X$-axis) and percent of correctly assembled (Y-axis), are shown for each genome. Metrics for the Assembly method are shown in circles and the SpaRC-hybrid method in stars. (C) Bar charts of biosynthetic gene clusters (BGCs) predicted from the Biocrust dataset. Here we directly compared the difference in predicted BGCs counts for major BGC classes between assembly with metaFlye and our SpaRC-hybrid approach with the same assembler.

Currently, SpaRC-hyrbid tends to produce a more fragmented assembly containing more small errors (mismatches, small indels). The most likely cause for this problem is under-clustering, as reads from the same genome were separated into different clusters. In the subsequent assembly step, each cluster does 


\section{CONFLICT OF INTEREST STATEMENT}

\section{AUTHOR CONTRIBUTIONS}

\section{FUNDING}

283 The work was supported by the National Natural Science Foundation of China (No. 61802246) and the 111

\section{DISCLAIMER}

No approval or endorsement of any commercial product by the National Institute of Standards and Technology is intended or implied. Certain commercial software, products, and systems are identified in this report to facilitate better understanding. Such identification does not imply recommendations or endorsement by NIST, nor does it imply that the software and products identified are necessarily the best available for the purpose.

\section{ACKNOWLEDGMENTS}

We thank the scientific computing group at Berkeley lab, especially Dr. Shawfeng "Shaw" Dong and Dr. Wei Feinstein for their support to run SpaRC on Lawrencium. We thank members of NERSC, for their support to run SpaRC on the Cori system.

\section{REFERENCES}

Arumugam, M., Raes, J., Pelletier, E., Le Paslier, D., Yamada, T., Mende, D. R., et al. (2011). Enterotypes of the human gut microbiome. nature 473, 174-180

Bertrand, D., Shaw, J., Kalathiyappan, M., Ng, A. H. Q., Kumar, M. S., Li, C., et al. (2019). Hybrid metagenomic assembly enables high-resolution analysis of resistance determinants and mobile elements in human microbiomes. Nature biotechnology 37, 937-944

Blin, K., Shaw, S., Steinke, K., Villebro, R., Ziemert, N., Lee, S. Y., et al. (2019). antismash 5.0: updates to the secondary metabolite genome mining pipeline. Nucleic acids research 47 , W81-W87

Breitwieser, F. P., Lu, J., and Salzberg, S. L. (2019). A review of methods and databases for metagenomic classification and assembly. Briefings in bioinformatics 20, 1125-1136 
bioRxiv preprint doi: https://doi.org/10.1101/2021.01.25.428115; this version posted January 26,2021 . The copyright holder for this preprint (which was not certified by peer review) is the author/funder. This article is a US Government work. It is not subject to copyright under 17 USC 105 and is also made available for use under a CCO license.

Lu, et al.

Metagenome Hybrid Clustering

304

[Dataset] Bremges, A. and McHardy, A. C. (2018). Critical assessment of metagenome interpretation enters the second round.

Bushnell, B., Rood, J., and Singer, E. (2017). Bbmerge-accurate paired shotgun read merging via overlap. PloS one 12, e0185056

Chin, C.-S., Alexander, D. H., Marks, P., Klammer, A. A., Drake, J., Heiner, C., et al. (2013). Nonhybrid, finished microbial genome assemblies from long-read smrt sequencing data. Nature methods 10, 563-569

Dmitry, Antipov, Anton, Korobeynikov, Jeffrey, S, et al. (2016). hybridspades: an algorithm for hybrid assembly of short and long reads. Bioinformatics

Eid, J., Fehr, A., Gray, J., Luong, K., Lyle, J., Otto, G., et al. (2009). Real-time dna sequencing from single polymerase molecules. Science 323, 133-138

Gurevich, A., Saveliev, V., Vyahhi, N., and Tesler, G. (2013). Quast: quality assessment tool for genome assemblies. Bioinformatics 29, 1072-1075

Hess, M., Sczyrba, A., Egan, R., Kim, T.-W., Chokhawala, H., Schroth, G., et al. (2011). Metagenomic discovery of biomass-degrading genes and genomes from cow rumen. Science 331, 463-467

Hofmeyr, S., Egan, R., Georganas, E., Copeland, A. C., Riley, R., Clum, A., et al. (2020). Terabase-scale metagenome coassembly with metahipmer. Scientific reports $10,1-11$

Hugenholtz, P. and Tyson, G. W. (2008). Metagenomics. Nature 455, 481-483

Kang, D. D., Froula, J., Egan, R., and Zhong, W. (2015). Metabat, an efficient tool for accurately reconstructing single genomes from complex microbial communities. Peerj 3, e1165

Kang, D. D., Li, F., Kirton, E., Thomas, A., Egan, R., An, H., et al. (2019). Metabat 2: an adaptive binning algorithm for robust and efficient genome reconstruction from metagenome assemblies. PeerJ 7, e7359

Kolmogorov, M., Bickhart, D. M., Behsaz, B., Gurevich, A., Rayko, M., Shin, S. B., et al. (2020). metaflye: scalable long-read metagenome assembly using repeat graphs. Nature Methods 17, 1103-1110

Koren, S. and Phillippy, A. M. (2015). One chromosome, one contig: complete microbial genomes from long-read sequencing and assembly. Current opinion in microbiology 23, 110-120

Koren, S., Walenz, B. P., Berlin, K., Miller, J. R., Bergman, N. H., and Phillippy, A. M. (2017). Canu: scalable and accurate long-read assembly via adaptive k-mer weighting and repeat separation. Genome research $27,722-736$

Li, D., Liu, C. M., Luo, R., Kunihiko, S., and Tak-Wah, L. (2015). Megahit: an ultra-fast single-node solution for large and complex metagenomics assembly via succinct de bruijn graph. Bioinformatics 31, 1674-1676

Li, K., Lu, Y., Deng, L., Wang, L., and Wang, Z. (2020). Deconvolute individual genomes from metagenome sequences through short read clustering. Peerj 8, e8966

Logsdon, G. A., Vollger, M. R., and Eichler, E. E. (2020). Long-read human genome sequencing and its applications. Nature Reviews Genetics , 1-18

Medema, M. H., Kottmann, R., Yilmaz, P., Cummings, M., Biggins, J. B., Blin, K., et al. (2015). Minimum information about a biosynthetic gene cluster. Nature chemical biology 11, 625-631

Mikheenko, A., Saveliev, V., and Gurevich, A. (2016). Metaquast: evaluation of metagenome assemblies. Bioinformatics 32, 1088-1090

Nayfach, S., Roux, S., Seshadri, R., Udwary, D., Varghese, N., Schulz, F., et al. (2020). A genomic catalog of earth's microbiomes. Nature Biotechnology , 1-11

Nurk, S., Meleshko, D., Korobeynikov, A., and Pevzner, P. A. (2017). metaspades: a new versatile metagenomic assembler. Genome research 27, 824-834 
Parks, D. H., Rinke, C., Chuvochina, M., Chaumeil, P.-A., Woodcroft, B. J., Evans, P. N., et al. (2017). Recovery of nearly 8,000 metagenome-assembled genomes substantially expands the tree of life. Nature microbiology 2, 1533-1542

Raghavan, U. N., Albert, R., and Kumara, S. (2007). Near linear time algorithm to detect community structures in large-scale networks. Physical review E 76, 036106

Schneider, G. F. and Dekker, C. (2012). Dna sequencing with nanopores. Nature biotechnology 30, 326-328

Sczyrba, A., Hofmann, P., Belmann, P., Koslicki, D., Janssen, S., Dröge, J., et al. (2017). Critical assessment of metagenome interpretation-a benchmark of metagenomics software. Nature methods 14 , 1063

Sevim, V., Lee, J., Egan, R., Clum, A., Hundley, H., Lee, J., et al. (2019). Shotgun metagenome data of a defined mock community using Oxford Nanopore, PacBio and Illumina, technologies. Scientific data 6 , 285-293

Shi, L., Meng, X., Tseng, E., Mascagni, M., and Wang, Z. (2018). Sparc: scalable sequence clustering using apache spark. Bioinformatics 35, 760-768

Stewart, R. D., Auffret, M. D., Warr, A., Walker, A. W., Roehe, R., and Watson, M. (2019). Compendium of 4,941 rumen metagenome-assembled genomes for rumen microbiome biology and enzyme discovery. Nature biotechnology 37, 953

Tyson, G. W., Chapman, J., Hugenholtz, P., Allen, E. E., Ram, R. J., Richardson, P. M., et al. (2004). Community structure and metabolism through reconstruction of microbial genomes from the environment. Nature 428, 37-43

Van Goethem, M. W., Osborn, A. R., Bowen, B., Andeer, P. F., Swenson, T. L., Clum, A., et al. (2021). Long-read metagenomics of soil communities reveals phylum-specific secondary metabolite dynamics. bioRxiv doi:10.1101/2021.01.23.426502

White, R. A., Bottos, E. M., Chowdhury, T. R., Zucker, J. D., Brislawn, C. J., Nicora, C. D., et al. (2016). Moleculo long-read sequencing facilitates assembly and genomic binning from complex soil metagenomes. MSystems 1

Wick, R. R., Judd, L. M., Gorrie, C. L., and Holt, K. E. (2017). Unicycler: resolving bacterial genome assemblies from short and long sequencing reads. PLoS computational biology 13, e1005595

Wommack, K. E., Bhavsar, J., and Ravel, J. (2008). Metagenomics: read length matters. Applied and environmental microbiology 74, 1453-1463

$\mathrm{Xu}, \mathrm{J}$. (2006). Invited review: microbial ecology in the age of genomics and metagenomics: concepts, tools, and recent advances. Molecular ecology 15, 1713-1731

Zimin, A. V., Marçais, G., Puiu, D., Roberts, M., Salzberg, S. L., and Yorke, J. A. (2013). The masurca genome assembler. Bioinformatics 29, 2669-2677 


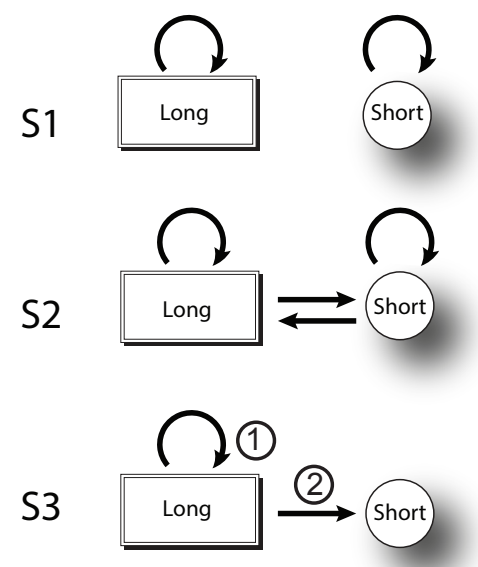

Label Propogation Schemes
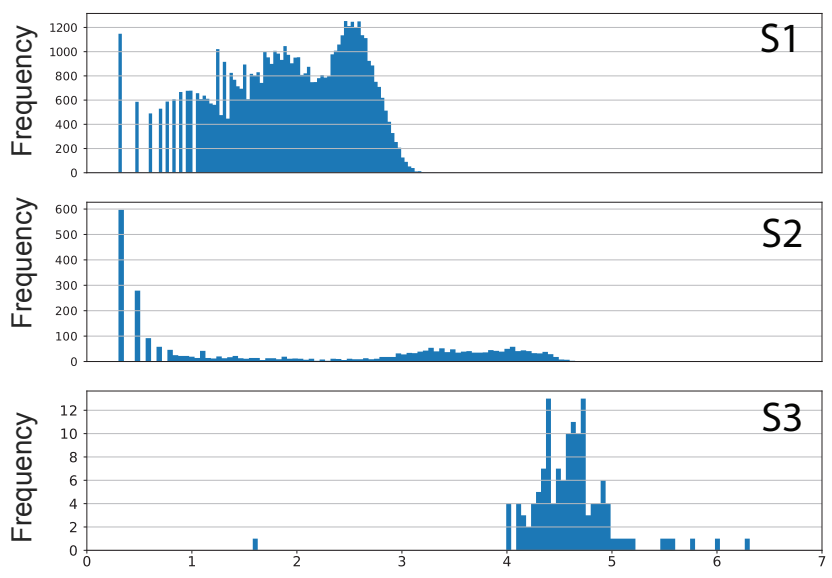

Cluster Size $(\log 10)$

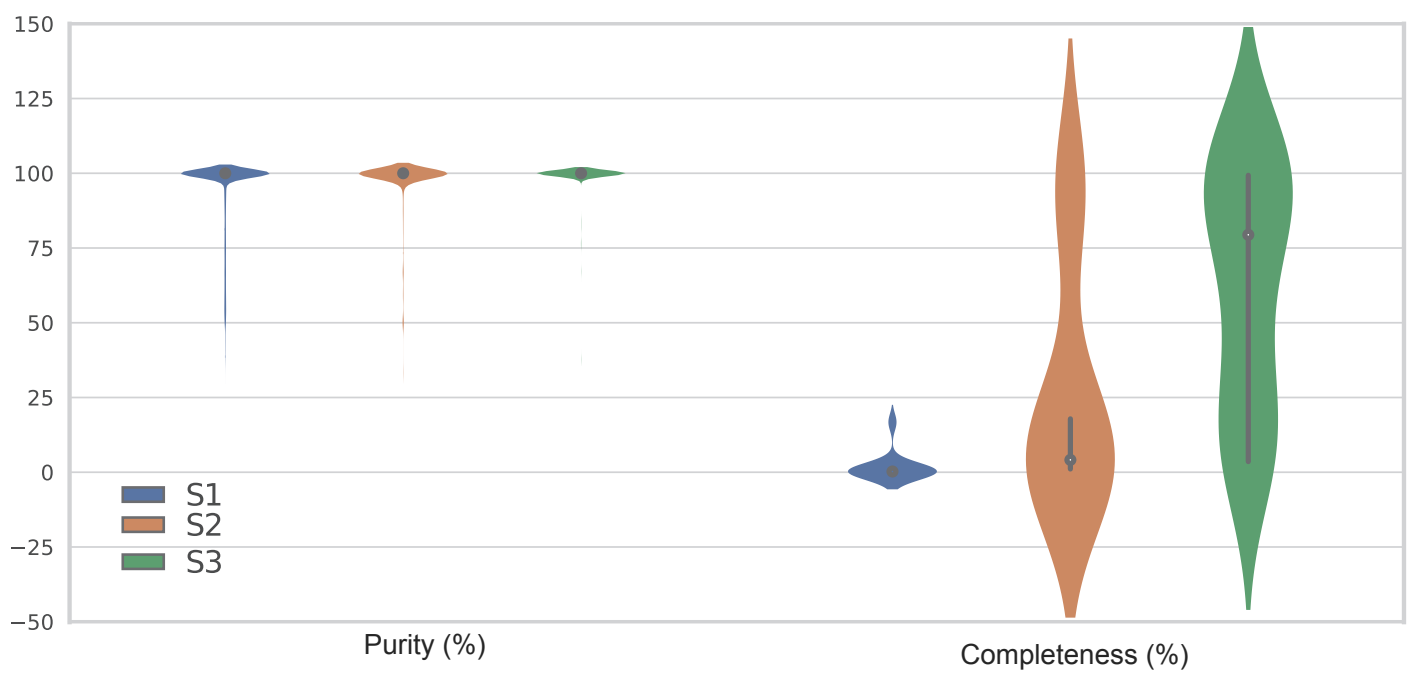


-0) mean cluster size (k)

- ๑) \#reads (M)

- (2) \#clusters

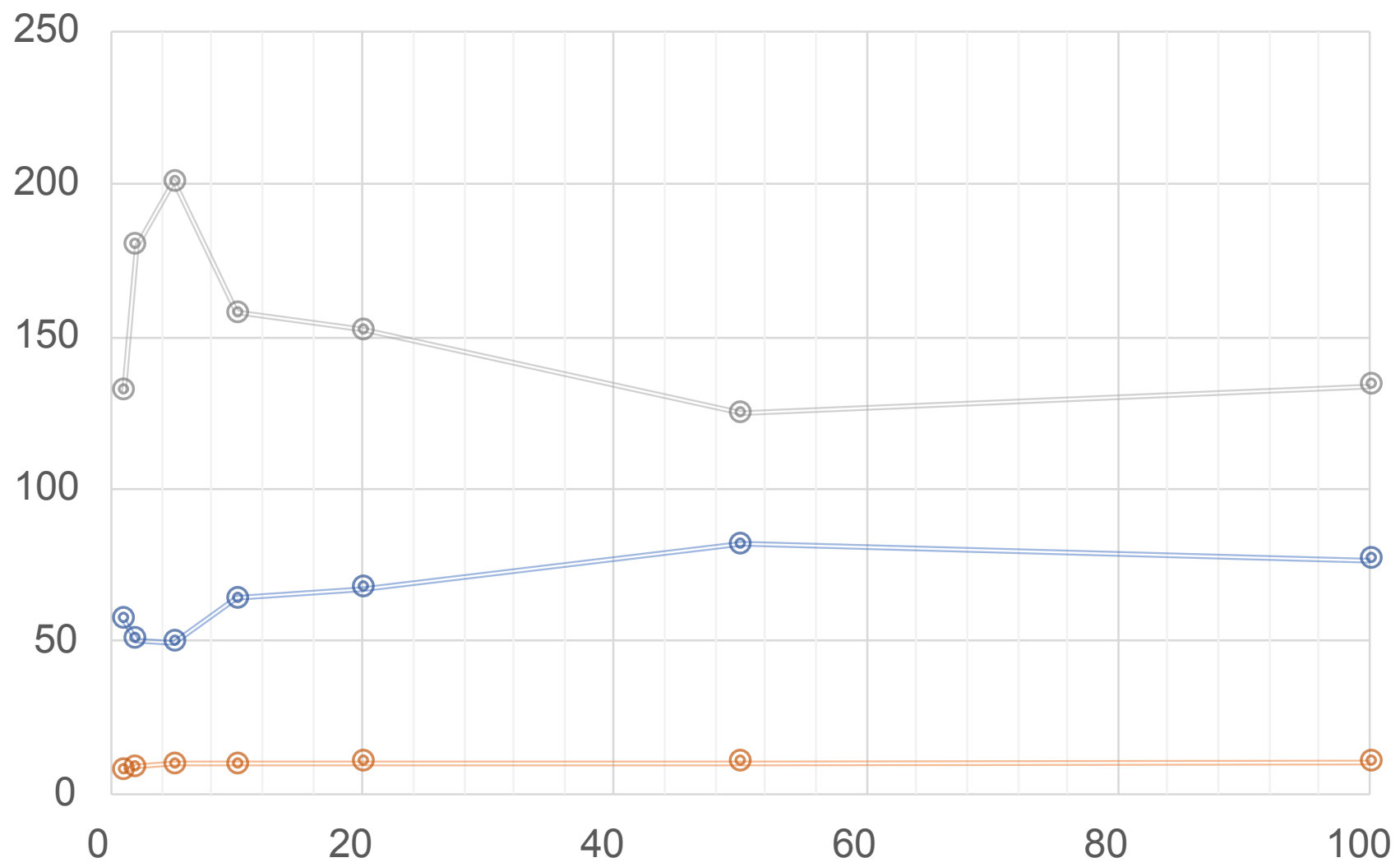

Percent of long reads used (\%) 

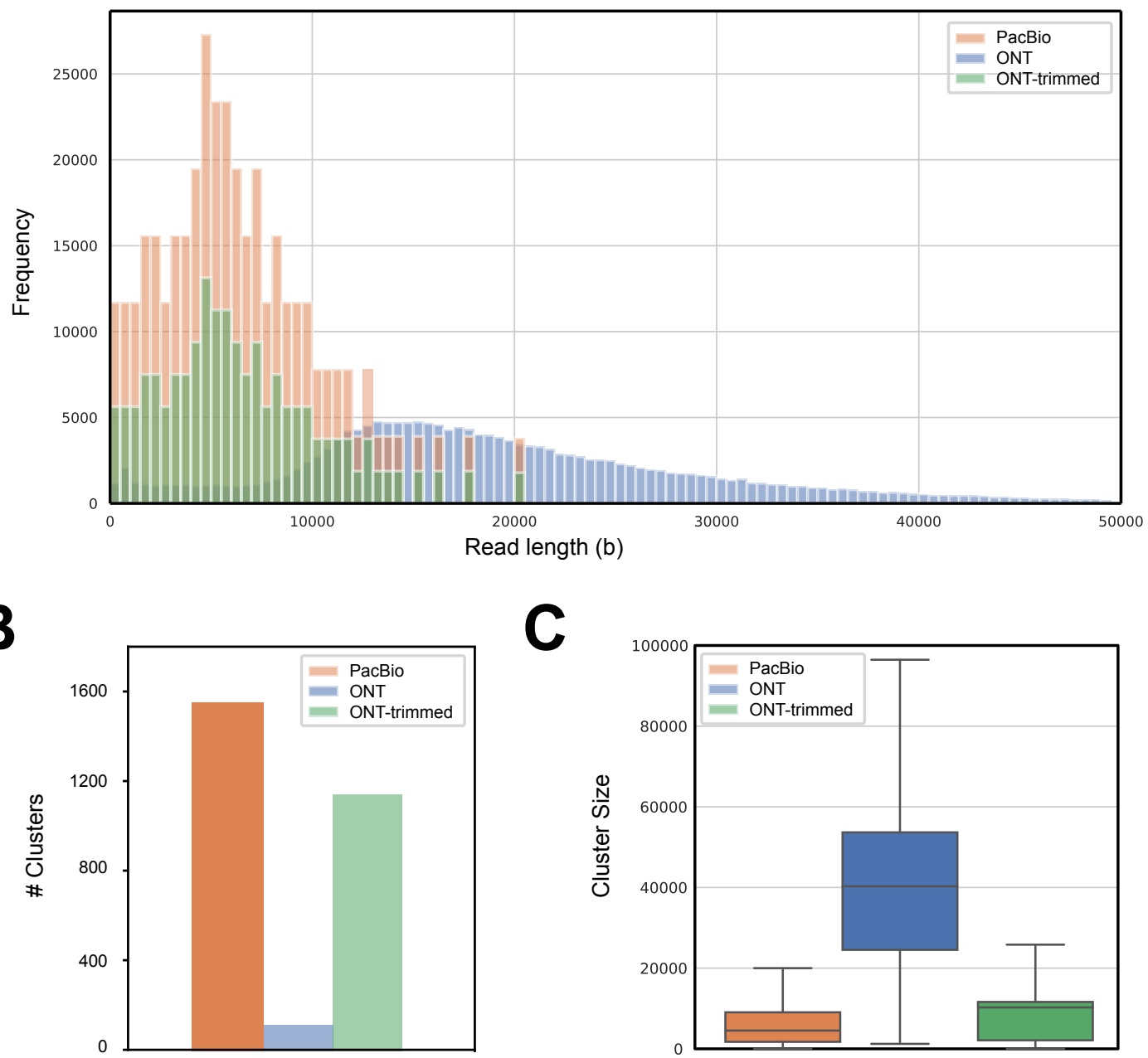
B

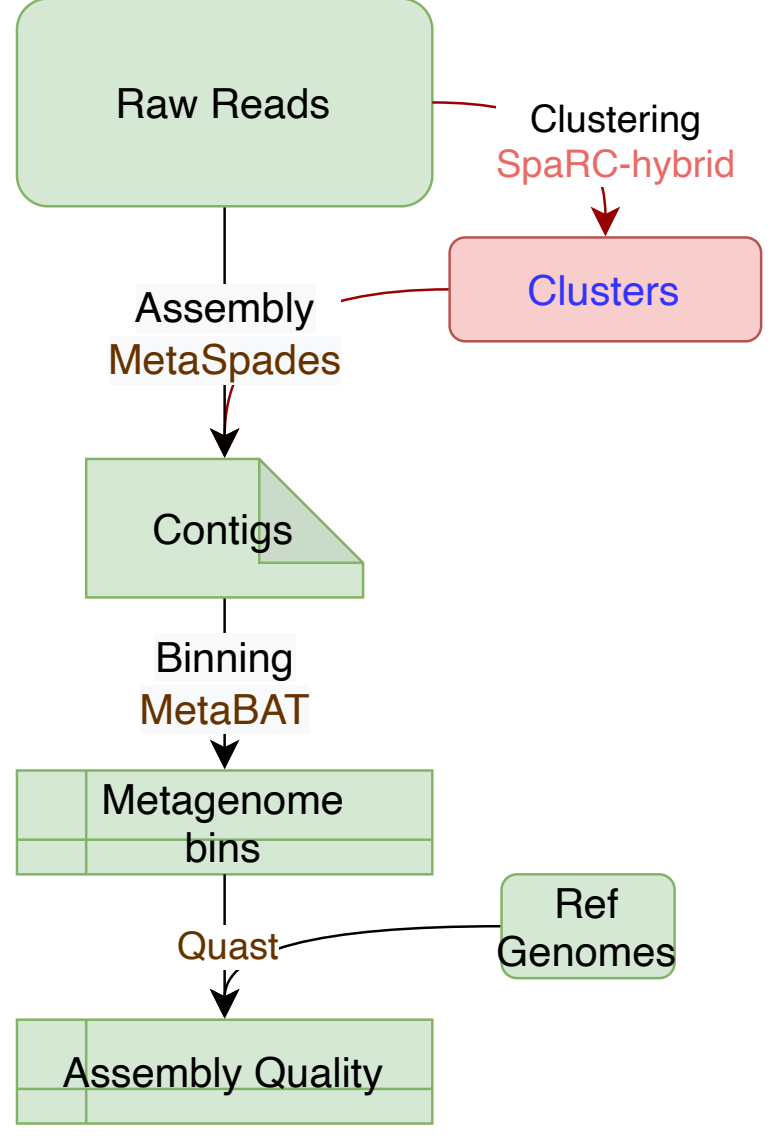

Assembly vs SpaRC_hybrid
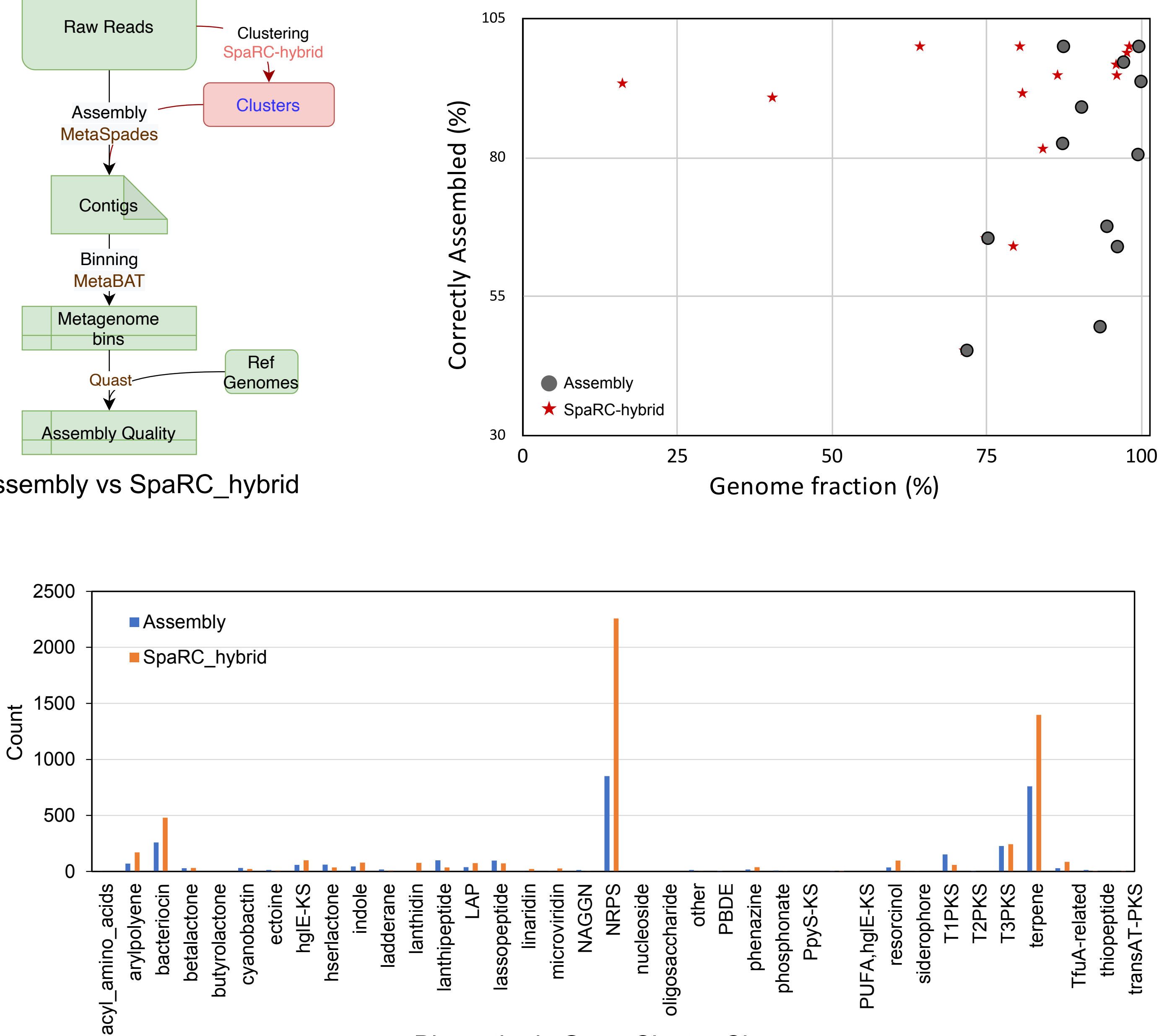

Biosynthetic Gene Cluster Class 